\title{
Fragmentation des Territoires Urbains et Développement Soutenable: Un rôle central de régulation politique pour les villes et les régions urbaines
}

\section{Claude Jacquier*}

\begin{abstract}
R Lésumé: L'article proposé prend appui sur plusieurs travaux de recherche réalisés depuis une vingtaine d'années en Europe et ailleurs (Amérique du Nord, Afrique) sur le thème de la fragmentation sociale et spatiale des villes et sur les politiques de développement soutenable mises en œuvre au sein des divers pays. La première partie de l'article essaie de tirer quelques enseignements des recherches conduites en Europe tant en ce qui concerne ces territoires que les politiques mises en œuvre. La seconde partie met en évidence quelques-unes des pistes de recherche à poursuivre pour essayer de contribuer à une meilleure prospective possible de ces territoires et de ces politiques à l'échelle européenne. En particulier, concernant l'Europe et ses difficultés à tenir les engagements de Lisbonne, cet article essaie de montrer comment, autour de ces enjeux et des approches intégrées de développement soutenable s'affirme le rôle politique nécessaire des villes et des régions urbaines dans la régulation des territoires que les Etats-membres ont eu, jusqu'ici, bien de la peine à leur reconnaître.
\end{abstract}

Mots-clés: Territoires, communautés, fragmentation sociale et spatiale, politiques intégrées de développement urbain soutenable, gouvernance, coproduction, coopération

\section{Introduction}

A la lumière de quelques recherches européennes, ce texte a pour ambition de donner un éclairage sur la réalité de ces quartiers de ville qu'on dit en difficultés («deprived areas» en anglais) en questionnant les représentations figurée de ces territoires et les formulations des politiques qui les concernent. Généralement, les représentations contrastées utilisant des catégories rigides l'emportent sur une vision nuancée d'une réalité urbaine qui est pourtant si paradoxale. L'évolution des multiples constituants élémentaires des territoires urbains n'obéit pas à des déterminations causales assurées. On est souvent en présence de systèmes complexes dont les composantes interagissent entre elles selon des règles locales non linéaires. Ainsi, tendances et contre-tendance coexistent et il est difficile a priori de dire ce qu'elles génèreront et ce qui en émergera au sens des théories de la complexité (Zwirn, 2006).

La figure de la fragmentation sociale et spatiale est aujourd'hui, en Europe, la représentation la plus largement acceptée pour rendre compte de l'évolution des villes et de la hiérarchisation de leurs territoires avec d'un côté les «gated communities» et de l'autre les quartiers «délaissés». Certains y voient un processus récent en voie d'accélération et d'approfondissement mettant en péril la cohésion sociale des villes et pouvant menacer les fondements démocratiques de nos sociétés. D'autres soulignent que cette fragmentation est ancienne, quasiment consubstantielle au fait urbain et qu'il

\footnotetext{
* Directeur de recherche au CNRS, Unité Mixte de Recherche TRIANGLE, ECOLE NORMALE SUPERIEURE - LETTRES ET SCIENCES HUMAINES, INSTITUT D'ETUDES POLITIQUES, UNIVERSITE LOUIS LUMIERE, LYON, 15, parvis escartes BP.7000, 69342 Ly॰n Cedex 07. Contacto: Claude.jacquier7@wanadoo.FR
} 
convient de s'accommoder de ce processus car il s'autorégule (Machiavel, 1952).

Assez fréquemment en Europe, l'analyse de cette réalité urbaine fragmentée se heurte au moins à deux écueils de la représentation. D'une part, une représentation euphémisée de la réalité qui confine au politiquement correcte: quartiers «en difficultés», quartiers «sensibles», parfois, tout simplement on les désigne par les termes de «quartiers»ou de «zones» sans autres attributs, ce silence étant tout à fait éloquent. Cette représentation évacue du discours une conflictualité, pourtant toujours bien présente, mais dissimulée, voire refoulée. Aujourd'hui, il n'est en effet plus guère possible de parler de la ville en termes de luttes de groupes ou de classes pour l'appropriation des territoires et des instances de pouvoir. Le second écueil tient à une représentation souvent caricaturale de ces territoires. Assez fréquemment, ces espaces sont stigmatisés par l'attribution de qualificatifs qui renvoient soit à des figures historiques de la mauvaise réputation (cf. «Chicago», ghettos, quartiers enclavés), soit à certaines caractéristiques d'une partie des gens qui les peuplent (quartiers d'immigrés, quartiers de chômeurs, quartiers de délinquants, etc.), tout cela relevant de pathologies qui nécessitent des interventions radicales (assainissement, interventions sécuritaires, éradication, démolition, etc.). Pour ma part, je préfère utiliser la dénomination «territoires délaissés» de préférence à celle de quartiers sensibles (euphémisme) ou de quartiers à problèmes (caricature) en référence à la notion que l'on utilise pour désigner ces espaces résiduels (dans les exploitations agricoles, le long des routes, des chemins de fer, etc.), espaces qui ne sont pas sans valeurs et qui souvent peuvent recéler des richesses et des opportunités de développement.

Ces représentations du monde urbain ont du mal à rendre compte de la réalité paradoxale de la ville. Les métropoles européennes, comme d'autres dans le monde, sont en effet, tout à la fois, l'ombilic du monde et sa périphérie appauvrie. On est plutôt en présence d'enclavements branchés et de cœurs de diasporas, les territoires délaissés étant en effet mieux branchés sur le monde que d'autres apparemment mieux achalandés. Les divers morceaux de ville peuvent être aussi qualifiés par des oxymores. Ils ne sont pas une chose OU son contraire, mais à la fois une chose ET son contraire. Tout l'enjeu d'une analyse un peu sérieuse réside dans la capa- cité de surmonter l'obstacle de ces deux types de représentation et de penser la réalité complexe et donc paradoxale de la ville

Dans cet article, nous nous limiterons à reprendre quelques uns des résultats de travaux de recherche portant sur les territoires urbains délaissés ainsi qu'aux politiques, programmes et projets qui les prennent pour objet sachant que la réflexion sur le rôle politiques des villes devrait prendre en compte une réalité urbaine bien plus large. La première partie de l'article essaie de tirer quelques enseignements des recherches conduites en Europe, tant en ce qui concerne ces territoires que les politiques mises en œuvre. Dans la seconde partie, nous indiquerons quelques-unes des pistes de recherche déjà engagées et à poursuivre pour essayer de contribuer à une meilleure prospective possible de ces territoires et de ces politiques à l'échelle européenne. En particulier, concernant l'Europe et ses difficultés à tenir les engagements de Lisbonne, nous essayerons de montrer comment, autour de ces enjeux et des approches intégrées de développement soutenable s'affirme le rôle politique central des villes et des régions urbaines que les Etats-membres ont eu jusqu'ici bien de la peine à leur reconnaître.

\section{Quelques Enseignements Concernant les Territoires et les Politiques Urbaines}

\section{La redécouverte de la complexité urbaine: «faire avec la ville».}

Les sociétés humaines ont connu et connaissent toujours de grands bouleversements. Pour en rendre compte on fait fréquemment référence à des notions générales censées avoir raison de tout: mondialisation, globalisation, marché, complexité croissante, etc. Ces références ont sans doute quelque intérêt mais, pour autant, ont-elles une pertinence explicative plus grande que celles qui étaient mobilisées en d'autres temps pour rendre compte de la réalité: le capital, le prolétariat, l'exploitation, le bien et le mal ou encore... Dieu. On peut en douter! Le processus de mondialisation est multiséculaire et s'il s'est accéléré s'appuyant sur de nouvelles technologies, a-t-il vraiment changé de nature? Par ailleurs, le monde est-il devenu véritablement plus complexe ou bien découvrons-nous enfin cette complexité que nous ne savions 
ou ne voulions pas voir jusqu'ici. Je penche pour les seconds termes de ces alternatives.

Dans les pays de vieille industrialisation, la période d'intense urbanisation des décennies de l'après guerre a fait place à une phase de croissance ralentie des villes au sein de leurs traditionnels périmètres d'urbanisation. La construction de logements est un bon indicateur de cette évolution. Dans la plupart des pays européens, le rythme annuel de construction de logement pour 1000 habitants a été quasiment divisé par deux entre 1970 et $2000^{1}$. Dans le périmètre classique des villes européennes, ce ralentissement a encore été plus marqué (pénurie de terrains constructibles à un coût foncier acceptable, réglementations plus strictes, diminution du rythme de construction, diminution de la taille des opérations). Le processus d'urbanisation traditionnel, à savoir un processus de concentration de populations venues d'ailleurs (ruraux, immigrés) sur un territoire délimité a touché à son terme. Depuis deux décennies, on assiste à une inversion du processus: dépopulation des villes centre, dissémination de la ville dans sa lointaine périphérie, recyclage des espaces anciennement urbanisés, renouvellement démographique sur une base interne. De machines à attirer, à capter et à concentrer des populations et des ressources sur des territoires densifiés à l'intérieur de frontières assez bien délimitées (cf. le modèle de la ville fortifiée), les villes sont devenues des régions urbaines proliférantes qui redistribuent de manière sélective des populations entre leur centre traditionnel et les lointaines périphéries (cf. le concept italien de la «città diffusa») (voir schéma ci-dessous).

Ces mutations de la réalité urbaine et ce renversement des représentations questionnent aussi les acteurs et les organisations capables de mettre en œuvre de telles stratégies de développement soutenable ainsi que les principes d'action politique pourtant admis de longue date. Il en est, en particulier, de la conception machiavélienne, puis hobbesienne du politique. Ainsi selon Hobbes, la régulation politique interne des sociétés s'est construite face à cet espace extérieur sans limite et menaçant, celui de l'étranger, de l'infidèle ou du barbare. Pour sortir de la guerre de tous contre tous, les hommes ont dû conclure un pacte définissant un espace à l'intérieur duquel ils ont renoncé à exercer leur violence. En échange de cette renonciation, ils ont pu alors se doter d'un pouvoir politique leur garantissant la sécurité (Hobbes, 1921). De cette différence entre les modes internes et externes de gestion de la violence a découlé la différence de fonctions entre la police et l'armée et d'une certaine manière la répartition des rôles sociaux (Viveret, 1993). Mais que devient cette conception hobbesienne du politique encore largement acceptée partout lorsque, tous les enjeux se redistribuant sur de vastes régions-archipels urbains, cette distinction entre l'intérieur et l'extérieur perd de sa netteté et de sa pertinence, dès lors que les frontières traversent les territoires urbains traditionnels? N'est-ce pas ce modèle interne-externe qui s'est aussi effondré avec les tours du World Trade Center, aux Etats-Unis, un certain 11 septembre, sous les attaques en provenance de l'intérieur du territoire américain?

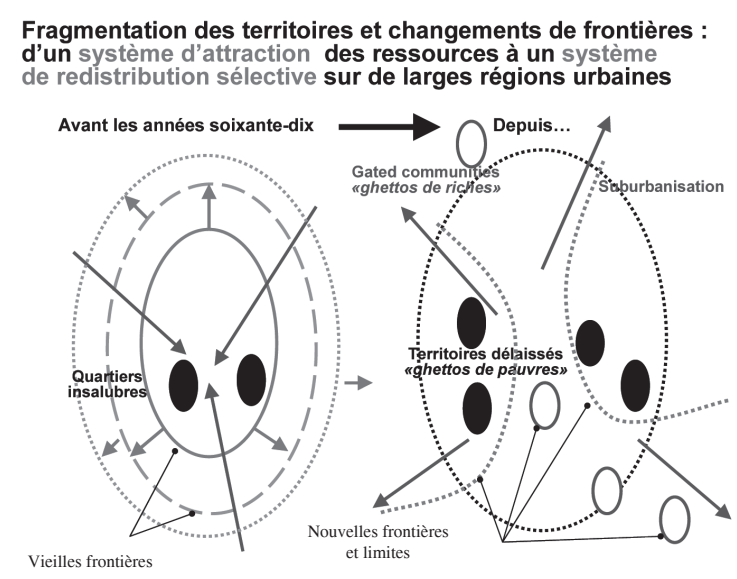

Avec la mutation des enjeux et ces changements de point de vue sur la ville, on est passé d'une approche centrée sur le «hardware» urbain à une approche de plus en plus focalisée sur le «software» social et sur le recyclage des territoires de la ville. Ainsi, la plupart des nouvelles politiques intégrées de développement urbain soutenable recensées en Europe, portent des dénominations composites dans lesquelles, soit le qualificatif social est explicitement retenu (Ville sociale en Allemagne, Impulsion social en Flandres, développement social

\footnotetext{
${ }^{1}$ Cette situation propre aux anciens pays industrialisés n'est en rien représentative des tendances qui prévalent dans le reste du monde et qui fait que désormais la moitié de la population de la planète est urbanisée. Ainsi, à son rythme actuel de construction annuelle, la Chine construira dans les 10 ou 12 prochaines années l'équivalent de la totalité du stock de logements existant sur le territoire des pays de l’Union européenne.
} 
en France, renouveau social aux Pays-Bas), soit il est fait mention de processus de recyclage urbain (programme pour les grandes villes en Belgique et aux Pays-Bas, programme de renouvellement urbain au Danemark, politique de la ville et renouvellement urbain en France, programmes de récupération urbaine et de développement durable des territoires en Italie, etc.).

Tout cela a conduit à une mutation des approches urbaines. On est ainsi passé d'une période caractérisée par le «faire la ville» (urbanisation) à une période où le «faire avec la ville» (urbanité) est devenu dominant. Le «faire la ville» reposait sur des processus d'urbanisation «productivistes», relativement frustes, se déroulant sur des sites «vierges» dont les caractéristiques sont alors niées (urbanisation massive des premières ceintures agricoles à la périphérie des villes, opérations de démolition et de rénovation des quartiers anciens), mis en œuvre par une autorité publique centrale, selon des procédures linéaires, sans guère de chevauchement des responsabilités politiques ou techniques. En revanche, le «faire avec la ville» dans lequel nous sommes entrés depuis deux décennies, consiste en une prise en compte beaucoup plus précautionneuse de la complexité des territoires urbains qui se manifeste souvent par une reconquête des espaces anciennement urbanisés (restauration immobilière, requalification, renouvellement et recyclage urbains, récupération des friches industrielles) exigeant de composer avec les territoires tels qu'ils existent, avec les populations, les institutions, les organisations et les acteurs qui y sont localisés ou qui y travaillent.

Ce passage du «faire la ville» au «faire avec la ville» repose sur une mutation du mode de développement urbain qui doit être accompagné par une profonde évolution des systèmes de gouvernance, des organisations professionnelles et des manières de faire. Or, le passage d'un mode de production et d'organisation à un autre, qui apparaît quasiment comme son opposé, ne se décrète pas. En la matière, en effet, il ne s'agit pas de substituer un produit urbain à un autre, mais il s'agit bien de transformer les processus de production eux-mêmes et donc des comportements. On ne passe pas brutalement d'une production sectorisée, relativement standardisée, opérant selon des procédures linéaires et faisant appel à des opérations successives clairement identifiées à un processus de production qui fait appel à la simultanéité et à l'interaction des interventions d'une pluralité d'acteurs aux savoir-faire fort différents, mais coopérant de manière régulée pour atteindre un objectif mutuellement négocié.

\section{La fragmentation des territoires urbains: quels enseignements pour la prospective urbaine?}

Le survol des villes et des quartiers montre la diversité des réalités urbaines en Europe, entre les villes et en leur sein. Les territoires délaissés présentent une assez grande diversité de localisations et de morphologies. Il peut s'agir de quartiers anciens de centre-ville, de faubourgs ou de quartiers populaires du $19^{\text {ème }}$ siècle, de cités ouvrières ou d'entreprises, de grands ensembles d'habitation construits dans les années de l'après-guerre ou encore de quartiers pavillonnaires plus récents. Parfois, on trouve sous ce label des bidonvilles et des zones d'habitat précaire. Les territoires concernés sont soit des quartiers d'habitat social du type grands ensembles des années 1960-70 (Milan, Gorbals et Castlemilk à Glasgow, Marzahn à Berlin, la Mina à San Adria de Besos, la muraille de Chine à St Etienne, Vaulx-en Velin et la Duchère Grand Lyon, etc.), soit des parties de centres historiques ou des quartiers anciens dégradés (Berlin, Birmingham, Budapest, Torino). Certains sont des zones mixtes habitat activités (Den Haag, Amsterdam Rotterdam), des quartiers ouvriers du $19^{\text {ème }}$ siècle ou du début du $20^{\text {ème }}$, des zones pavillonnaires, etc. Ainsi, en Europe, sur les 114 sites sélectionnés sur la période 1994-1999 du PIC «Urban», 55 étaient des quartiers anciens souvent localisés dans les centres historiques des villes (c'est le cas notamment en Espagne et en Italie), 12 étaient des grands ensembles ou assimilés (dont 6 en France), le reste des quartiers se partageant entre des sites mixtes habitat-activité, des sites de faubourg (tissu urbain des quartiers ouvriers de la fin du xix ${ }^{\text {ème }}$ siècle), des cités ouvrières du début du siècle, des sites pavillonnaires ou encore de l'habitat spontané ou précaire (Grèce, Portugal). Nous sommes donc loin d'un modèle standard.

Cette diversité est tout d'abord à référer est à des histoires différentes et à des constructions socio-démographiques singulières liées à l'inscription spécifiques des villes et des Etats-nations dans la dynamique d'expansion économique mondiale. 
Si les villes de l'Union Européenne ont à peu près toutes été construites sur des modèles similaires (cf. la commune médiévale fortifiée pour certaine, la ville industrielle pour d'autres), elles ont connu des fortunes diverses liées notamment aux mutations industrielles de la fin du $19^{\text {ème }}$ siècle, à l'exode rural et au boom de l'urbanisation du $20^{\text {ème }}$ siècle. Ces mutations ne sont pas toutes survenues au même moment et n'ont donc pas eu les mêmes conséquences morphologiques, architecturales et urbanistiques. Par ailleurs, ces villes sont localisées dans des Etats-nations qui se sont inscrits diversement dans le processus de mondialisation de l'économie au cours de l'histoire. En particulier, certains pays ont été des empires coloniaux à des périodes plus ou moins lointaines (Portugal et Espagne, puis Pays-Bas, Royaume-Uni, France et dans une moindre mesure Belgique). Ces épisodes ont laissés des traces, tout d'abord dans les villes portuaires, puis plus tard dans l'ensemble des villes engagées dans la logique de l'industrialisation, par l'appel massif à la main d'œuvre immigrée (cf. l'histoire de l'esclavage, de la colonie et de l'immigration). D'autres ont noués des relations particulières, souvent conflictuelles, intra-européennes (cf. l'Allemagne par rapport aux pays de l'Europe centrale et de l'Est, à l'ex-Yougoslavie ou à la Turquie). Tout cela a généré des configurations particulières des territoires urbains.

Cette diversité est aussi liée aux actuels mécanismes de fragmentation sociale et spatiale des villes. Par exemple, certains pays n'ont pas connu, ou alors de manière sporadique, la construction de grands ensembles de logement social dans les années cinquante et soixante. C'est un phénomène particulier à certains pays de l'Europe de l'ouest qui a de curieuses similitudes en Europe centrale et de l'est. Dans les pays qui ont vu la construction de ces quartiers champignon, leur localisation est d'ailleurs assez diverse: en centre urbain (innercity), notamment au Royaume Uni (Glasgow Londres, Manchester) et parfois en France à la suite de la politique de rénovation urbaine des années soixante; dans les premières couronnes urbaines périphériques, comme c'est le cas de l'Allemagne (Berlin, Hambourg), de l'Espagne (Barcelone, Madrid), de la France (Paris, Lyon, St Etienne), de l'Italie (Turin, Milan, Naples), des Pays-Bas
Amsterdam, Rotterdam), du Royaume Uni (Glasgow), mais aussi dans les nouveaux pays membres. Dans certains cas, notamment au nord et au centre de l'Europe, ce processus d'urbanisation a reposé sur l'intervention active de la puissance publique et a pris la forme de la construction de logements locatifs publics alors que dans l'Europe du sud, il a été l'œuvre de promoteurs et de constructeurs privés pour des logements destinés à l'accession à la propriété ${ }^{2}$. Dans les nouveaux Etats-membres, ces grands ensembles sont restés jusqu'à une époque récente le lieu d'habitat des classes dirigeantes ou moyennes. La place et le rôle de ces grands ensembles dans l'organisation du marché urbain sont tout aussi divers. Certains de ces quartiers ayant apparemment les même caractéristiques architecturales et urbaines ont connu une rapide dévalorisation patrimoniale, sociale et surtout symbolique alors que d'autres se sont maintenus en bonne place dans la hiérarchie des formes urbaines, accueillant encore aujourd'hui des populations de classes moyennes, voire supérieures. Parfois, crise de l'offre de logement aidant et renchérissement brutal du prix du logement en accession à la propriété ou en location, certains de ces grands ensembles, après avoir été délaissés, font l'objet d'une forte demande de la part de nouvelles classes moyennes ou de jeunes ménages. Dans certaines villes particulièrement «branchées», on constate même un engouement pour ce modernisme architectural des années soixante jusqu'ici tant déprécié. Une hétérogénéité similaire peut être constatée dans les quartiers anciens et dans les centres des villes européennes. Dans certains pays, le début du mouvement de réhabilitation de l'habitat ancien et des phénomènes de «gentrification» date du début des années soixante-dix (France, Pays-Bas). Dans la plupart des villes européennes du nord, ce mouvement a été plus tardif. Dans certains pays, il n'en est encore qu'à ses balbutiements. Comme on peut le constater, la géographie des quartiers délaissés est très diverse en Europe. Elle dépend des caractéristiques de l'histoire des villes, de leur structure, de leur place et de leur rôle dans l'organisation du continent européen, dans ce temps long dont parlait Fernand Braudel (1980).

Comment peut-on qualifier ces territoires urbains et quels indicateurs peut-on utiliser pour

${ }^{2}$ Classement des pays selon l'importance décroissante du parc locatif public en 2004: 35\% aux Pays-Bas, 21\% au Royaume-Uni, 23\% en Autriche, $23 \%$ en Suède, $22 \%$ au Danemark, $17 \%$ en France, 14\% en Finlande, $7 \%$ en Irlande, $6 \%$ en Allemagne, $7 \%$ en Belgique, $4 \%$ en Italie, $3 \%$ au Portugal, $2 \%$ au Luxembourg, $1 \%$ en Espagne, $0 \%$ en Grèce. 
les décrire? L'appellation «quartiers en difficulté» (deprived areas) qui apparaît de prime abord comme une évidence au vu de certains indicateurs, n'est pas d'un maniement très aisé. Il est en effet très difficile d'appliquer ce qualificatif aux territoires qu'il nous a été donné de visiter au cours des vingt dernières années. De quel ordre de difficultés est-il question? Difficultés liées à l'état du bâti (qualité des matériaux, état de l'entretien)? Difficultés liées aux déperditions d'énergie et à la mauvaise maîtrise de l'énergie et aux nuisances environnementales? Difficultés liées aux formes architecturales et urbaines (bâtiments anciens, difficilement adaptables, rejet du modernisme architectural des années soixante, friches urbaines) ou encore à la position de ces territoires dans la hiérarchie des formes urbaines (état des dessertes et des voies de communication, localisation, proximité de zones polluées ou d'équipements nuisants...)? Difficultés liées à l'occupation sociale de ces quartiers (regroupement de populations peu fortunées, regroupement de communautés ethniques...)? Difficultés économiques (chômage, précarité, faiblesse des revenus, maque d'activités commerciales et de services? Difficultés institutionnelles (faiblesse des organisations civiques: associations d'habitants, associations culturelles et cultuelles, faible présence des services publics)? Difficultés politiques (faible nombre d'inscrits sur les listes électorales, fort niveau d'abstention, absence de droits de vote pour certains résidents, absence de systèmes de médiation)? Parfois ces traits caractéristiques sont contradictoires entre eux et il est donc délicat de construire un indicateur synthétique permettant de qualifier rapidement et sans conteste les phénomènes urbains rencontrés, cela malgré certaines tentatives (cf. le Z Score anglais à la fin des années quatre-vingt ou récemment l'audit urbain de l'Union Européenne). On a bien affaire à une pluralité de causalités et de déterminations, jouant le plus souvent de manière indépendante comme il est normal dans des situations contingentes.

Toutes ces difficultés se manifestent isolément ou simultanément: difficultés liées aux caractéristiques intrinsèques de ces territoires (qualité des lieux) ou difficultés relatives liées à la place que ces territoires occupent dans la hiérarchie urbaine et qui fait qu'ils sont, à un moment donné, réellement ou symboliquement des lieux où se concentrent tous ceux que ne veulent pas côtoyer les autres urbains. En fait, les caractéristiques intrinsèques, architec- turales et urbaines des lieux ont peu de pertinence pour rendre compte du positionnement de ces territoires dans la hiérarchie des valeurs urbaines et il apparaît plus judicieux de considérer ce positionnement comme assigné par le fonctionnement et la structuration de la ville, de la métropole, voire de la région urbaine toute entière. Avec le mouvement de périurbanisation qui accentue les opportunités de fragmentation sociale et spatiale des villes, ces territoires-là jouent un rôle déterminé dans la fabrique urbaine ce que reconnaissent bien, par delà la différence des qualificatifs, les formulations qui ont été utilisées au fil du temps par divers auteurs: espaces d'accueil, espaces d'hébergement, espaces de survie, abcès de fixation, espaces de l'assignation à résidence, espaces de relégation, espaces de confinement (containment), ghettos, etc.

\section{Des politiques intégrées de développement urbain soutenable en construction.}

Les politiques urbaines mises en ouvre ici et là, au fil du temps, se présentent sous des formulations tout aussi différentes ce qui tient à diverses raisons qui s'enracinent dans l'histoire des politiques publiques des pays considérés. Au cours des deux dernières décennies, on a ainsi assisté, tout particulièrement en Europe, à une floraison de ces nouvelles approches, à côté d'initiatives classiques prises tant dans le domaine de la lutte contre la pauvreté et l'exclusion que dans le domaine de l'aménagement territorial et de l'environnement. En fait, aucune de ces approches n'a véritablement atteint cet horizon ambitionné du développement urbain soutenable, ni dans sa formulation, ni bien évidemment dans sa mise en œuvre. Chacune pourtant contribue à sa manière à la construction de ce référenciel des approches intégrées de développement urbain soutenable que prônaient les travaux du Carrefour des villes présidé par Jacques Delors en 1993, les communications de la Commission préparatoires du Forum de Vienne en 1998 (Union Européenne, 1997), approches recommandées à nouveau par la communication de la Commission en juin 2006 (à la suite de la réunion de Bristol pendant la présidence britannique) et par la charte de Leipzig en mai 2007 (présidence allemande) (Commission 2006, Présidence allemande 2007).

Sans prétendre à l'exhaustivité, on peut citer par ordre chronologique d'apparition, quelques- 
-unes des initiatives prises par les pays Européens en ce domaine, initiatives qui ont contribué à initier ces nouvelles approches intégrées ou qui s'en sont inspiré

- en France, la politique de développement social des quartiers (1982), puis la politique de développement social urbain (1988), enfin la politique de la ville (1992), la loi Solidarités et renouvellement urbain en 2000;

- aux Pays-Bas, le programme pour les zones accumulant des problèmes PCG (1985), le programme de renouveau social (1989), puis la politique pour les Grandes Villes (1993);

- en Angleterre et au Pays de Galles, le programme City Challenge (1990), la politique du Single Regeneration Budget (1993), la politique New Deal for Communities (1998) ou encore l'Écosse avec le programme New Life for Urban Scotland (1988);

- en Région flamande, le Fonds flamand pour l'intégration (1986), en Région Bruxelloise, les projets de Quartiers (1987) et, au niveau fédéral, la politique des grandes villes (2000);

- au Danemark, le Comité des initiatives urbaines (1994) et le programme pour l'amélioration des aires urbaines (1996);

- en Finlande, les projets pilotes pour l'amélioration des banlieues (1996);

- en Norvège, le programme pour les zones urbaines défavorisées (1997);

- en Suède, les projets pour la périphérie et la politique pour les aires métropolitaines (1997);

- en Rhénanie du Nord-Wesphalie, le programme de renouveau urbain (1993) suivi aujourd'hui, pour l'ensemble de l'Allemagne, par la politique pour la ville solidaire (Sozial Stadt) 1998;

- en Italie, les programmes de récupération urbaine et de développement soutenable des territoires (i programmi di ricupero urbano e di sviluppo sostenibile dei territori -PRUSST) (1998) et les contrats de quartier.

Depuis le milieu des années quatre-vingt-dix, ces politiques se sont multipliées au niveau national ou régional. Elles ont gagné en importance, relayées qu'elles étaient par l'Union Européenne qui a joué parfois un rôle d'initiateur (notamment dans les pays de l'Europe du sud) avec le lancement du programme d'initiative communautaire URBAN I en 1994 auquel le programme URBAN II a succédé pour la période 2000-2006. On peut imaginer qu'il en sera de même pour les nouveaux Etats-membres et pour les pays du bassin méditerranéen concernés par la programmation des fonds structurels pour la période 2007-2013 avec la volonté de renationaliser les initiatives en ce domaine à partir de l'acquis URBAN (Orientations Stratégiques Communautaires-OSC au niveau de la Commission, Cadre de Référence Stratégique National-CRSN au niveau national et Programmes Opérationnels-PO au niveau régional). Dans le rôle de dissémination des savoirs, des savoir-faire et des bonnes pratiques, il faut souligner ici le rôle précurseur de réseaux de ville tels que le Réseau Quartiers en Crise qui, de 1988 à 1994, a été une des pépinières de ces nouvelles pratiques en favorisant les échanges et la capitalisation entre les expériences locales. Malgré le souhait de voir ce réseau d'échange se généraliser au moment du lancement du programme URBAN 1 en 1994, il a fallu attendre 2003 pour que voit le jour le programme URBACT 1, programme d'échange, de capitalisation et de dissémination des approches intégrés de développement soutenable entre une centaine de villes européennes réunies au sein de réseaux thématiques. Sur la période 2007-2013, le programme URBACT 2 va démultiplier son action puisque plus de 400 villes européennes vont être concernées au sein de réseaux thématiques au sein desquels figureront à parité des villes de la zone Convergence (nouveaux Etats membres) et des villes de la zone Compétitivité régionale et emploi (anciens Etats membres). Cette action va être renforcée et approfondie puisqu'aux activités d'échange, de capitalisation et de dissémination vont être ajoutées des activités de coopération entre les villes membre des réseaux afin de leur permettre d'élaborer des programmes intégrés de développement soutenable (fonction d'ingénierie mutualisée et de labellisation des projets auprès des autorités régionales, nationales et européennes).

Les programmes recensés dans divers pays ne sont souvent que des esquisses des approches intégrées de développement urbain soutenable tant souhaitées. Ces programmes s'inscrivent dans de véritables stratégies visant la coproduction de 
nouvelles politiques urbaines à l'échelle européenne. Sans doute ces programmes sont encore prisonniers d'approches sectorielles ou sectorisées. Ainsi, les projets visant la transformation physique des territoires l'emportent encore souvent sur les projets à dimension sociale, économique et institutionnelle. Parfois, comme en France avec le programme de rénovation urbaine, on assiste à un retour à des mesures radicales de démolition. Tous ces programmes mettent néanmoins l'accent sur la notion de développement entendu comme nécessité de dépasser des approches trop sectorisées, ciblées avant tout sur les lieux (targeted approaches), souvent sur les gens à qui ils font une place plus importante dans leur mise en œuvre, mais qui délaissent par trop la dimension institutionnelle et les nécessaires réformes politico-administratives à mettre en œuvre. Malgré des formulations parfois stigmatisantes, les programmes recensés ci-dessus essaient de souligner les richesses que recèlent ces territoires délaissés et les opportunités qu'ils offrent pour construire des stratégies de développement. Ils mettent alors l'accent sur les gens, les groupes sociaux dans leur diversité et sur leur capacité à participer aux projets et aux programmes, voire d'en être les moteurs. Souvent ces programmes incluent des cycles de formation des résidents et des stratégies d'empowerment.

Dans cette dernière option, le chemin est long cependant pour passer d'un regard compatissant, dans le meilleur des cas à une pleine reconnaissance de ces résidents comme acteurs du développement. Il est en effet difficile pour les autorités, les promoteurs et les responsables de projets et de programmes, par delà la critique qui leur est faite de vouloir imposer leur pouvoir, de ne pas projeter sur ces territoires leur conception de ce que doit être un bon quartier et une bonne ville en accord avec les normes institutionnelles, les critères techniques et les principes financiers bien établis, définis d'en haut. Par leur origine sociale et leur culture, par leur formation, par les contraintes institutionnelles qui pèsent sur eux, ces responsables sont souvent dans la position de reproduire simplement ce qui a déjà été fait, ce qui est éprouvé et parfois, plus prosaïquement, ce qui permet d'assurer la rentabilité d'un projet. Une telle attitude est encore assez fréquente dans les programmes conduits par des pouvoirs centralisés (publics ou privés) et qui opèrent de manière descendante (top-down). Les objectifs de tels programmes sont en effet rarement explicités, les transformations physiques s'accompagnant obligatoirement de mutations dans le peuplement des territoires et dans les systèmes de régulation hérités du passé sans que cela soit affiché dans la formulation des programmes. A cela rien d'étonnant, l'ambiguïté est souvent souhaitable et surtout souhaité en politique. Toute politique, c'est sa nature, est construite sur des dimensions paradoxales (effets d'annonce, effets pervers, effets feed-back plus ou moins maîtrisés, etc.).

La rupture qu'ambitionnent les approches intégrées de développement soutenable avec de telles approches «top down» et avec les ambiguïtés de leurs formulations n'est pas chose aisée. La condition nécessaire de tels changements réside dans l'existence de communautés structurées, la structuration de coalitions socio-politiques locales et la construction de rapports de forces favorables. Les programmes qui y parviennent le mieux et qui, d'une certaine manière, innovent parce qu'ils tiennent compte pleinement des éléments constitutifs des territoires sont ceux qui échappent d'une certaine manière à la logique des procédures centralisées et homogénéisantes pour privilégier le «faire avec». Il existe ainsi de remarquables projets de régénération urbaine nés de dynamiques locales qu'aucune autorité publique centrale n'a cherché à initier. C'est souvent lorsqu'il n'existe pas de politique stéréotypée centrale ou plutôt, lorsque ces politiques centrales ont mis l'accent, non pas sur le respect strict des procédures (objectifs de maîtrise et de contrôle), mais seulement sur la mise en synergie des partenaires potentiels (politiques et programmes incitateurs et mobilisateurs, mise à disposition de moyens financiers et techniques ainsi que de compétences) qu'ont surgi de nouvelles approches, de nouvelles problématiques et de nouvelles conceptualisations. Souvent des domaines particuliers (éducation, santé, sécurité, situation de handicap et accessibilité etc.) dont la caractéristique principale est plus que d'autres d'appeler la coopération et la coproduction entre les acteurs de terrain ainsi que des professionnels ou des dirigeants associatifs ou communautaires ont été des facilitateurs et des vecteurs de telles mises en synergie. 


\section{Pistes de Recherche pour une Meilleure Prospective des Territoires et des Politiques Urbaines}

Une réflexion est certainement à poursuivre et à approfondir sur cette «alchimie» particulière concernant les diverses formes de coproduction et de coopération que nous venons d'évoquer. Cette alchimie est en effet de plus au cœur des transformations urbaines récentes et des mutations dans les «manières de faire» la ville qui ont émergé au cours des deux dernières décennies et qui ont beaucoup à voir avec les exigences et les impératifs du développement urbain soutenable.

Dans cette seconde partie de cet article, à partir des enseignements tirés des expériences passées à l'échelle européenne, nous allons essayer de dégager quelques pistes de recherche qui pourraient être suivie dans les années à venir en faisant coopérer les chercheurs et les praticiens au sein de programmes européens, voire transcontinentaux. Plusieurs domaines pourraient faire l'objet de travaux plus systématiques. Tout d'abord, il apparaît nécessaire d'approfondir l'analyse des territoires urbains et des communautés locales pour se doter d'outils plus adaptés permettant de mieux comprendre les processus de fragmentation sociale et spatiale à l'œuvre au sein des villes. L'accent devrait être mis en parallèle sur une analyse plus précise et plus prospective des politiques urbaines à l'heure où le continent européen doit faire face à de multiples défis identifiés par les engagements de Lisbonne et de Göteborg (mondialisation accélérée, concurrence des pays asiatiques dans les secteurs de très hautes technologie) face auxquels les villes et les régions urbaines ont une carte politique majeure à jouer.

\section{«Faire avec» les territoires et les communautés locales}

Les enseignements des travaux de recherche comparatifs et les échanges entre chercheurs et praticiens en montrant la complexité des réalités urbaines, suggèrent de nous «limiter» dans cet article à une réflexion ciblée uniquement sur les unités urbaines de base, ce qu'on appelle traditionnellement les quartiers et ce que nous assimilons dans nos recherches aux communautés locales dans la définition qu'en donne le monde anglophone (community). Les recherches en ce domaine doivent identifier de manière plus systémique les éléments constitutifs de ces territoires et explorer leurs transformations possibles afin de mettre en évidence ce qui peut en émerger à un niveau supérieur, à l'échelle du fonctionnement global de la ville et de la région urbaine.

Pour identifier les constituants élémentaires de ces territoires-communautés nous avons repris à notre compte une définition dans un sens qui permet une approche analytique de cette réalité. Ainsi, comme l'indiquait fort justement Jean-François Médard dans son travail majeur sur les communautés et le développement communautaire aux Etats-Unis, «une communauté est à la fois un endroit, des gens vivant en cet endroit, l'interaction entre ces gens, les sentiments qui naissent de cette interaction, la vie commune qu'ils partagent et les institutions qui règlent cette vie» (Médard, 1969).

Quelle que soit l'échelle à laquelle on se place, un territoire peut être considéré comme une combinatoire singulière de trois éléments en résonnance avec les trois piliers du développement soutenable (l'environnement, le social et l'économie):

- Ce territoire est tout d'abord un lieu géographique («place» en anglais) à savoir un espace physique, un endroit. Ce lieu n'a plus grand-chose de naturel, c'est un artefact, un «produit» et un «construit» social qui présente certaines caractéristiques particulières: un patrimoine, un type de bâti et d'ordonnancement urbain, un rapport à l'environnement, des distances, une échelle, etc.). Ce lieu est un «être-là» particulier (de l'ordre du «dasein»), non substituable à un autre et qui peut être transformé, amélioré et bonifié mais aussi dégradé et détruit. On peut distinguer deux dimensions du lieu (Berque, 1999), le lieu mesurable qui renvoie au topos aristotélicien et le lieu vécu, le chôra platonicien, cette seconde dimension étant caractérisé par ce que l'on appelle l'esprit du lieu (genius loci). Ce constituant du territoire renvoie à la dimension environnementale identifiée comme étant un des piliers du développement soutenable. 
- Ce territoire est ensuite constitué par des gens («people») qui vivent et/ou travaillent en ces lieux et qui contribuent à le façonner par leurs caractéristiques et leurs capacités individuelles et collectives, donc par les relations et les rapports qu'ils ont noués entre eux et avec ce lieu, de longue date, pour construire un capital humain, un capital social et un capital culturel. Parmi ces relations, il faut insister ici sur celui qui est, pourrait-on dire, à la base de toute forme communautaire et qui conditionne sa reproduction, à savoir le rapport femme-homme. Peu pris en considération et le plus souvent oublié par l'analyse et dans les programmes mis en œuvre, ce rapport de genre doit faire l'objet d'une grande attention tout particulièrement dans les territoires délaissés où on note souvent une sur-représentation des familles monoparentales dans lesquelles le chef de famille est une femme. L'ensemble de ces relations et de ces rapports entre les gens constitue ce que l'on appelle l'être et le vivre ensemble («mitsein»). Cette composante du territoire renvoie en quelque sorte au second pilier du développement soutenable, à savoir le social.

- Enfin, ce territoire est structuré par des institutions que ces gens se sont données ou qui s'imposent à eux. La notion d'institution doit être prise au sens large. Il s'agit à la fois des organisations économiques (entreprises, commerces ou services) et des institutions ayant pour fonction de réguler les rapports entre les gens, entre les gens et les lieux ou encore entre ces territoires et l'environnement externe. Ces institutions qui présente la caractéristiques commune d'être des systèmes régis par des conventions sont censées contribuer à la régulation de cet être et de ce vivre ensemble (Arendt 1983). Lorsque ces institutions sont uniquement constituées sur une base homogène qu'elle soit sociale, ethnique ou religieuse, le territoire ou la communauté tend à épouser la forme de ce que l'on qualifie généralement du nom de «ghetto». Cette troisième com- posante élémentaire du territoire renvoie au troisième pilier du développement soutenable, à savoir l'économie dès lors qu'on ne réduit pas celle-ci à sa seule acception productive et marchande.

\section{Territoires et communautés locales}

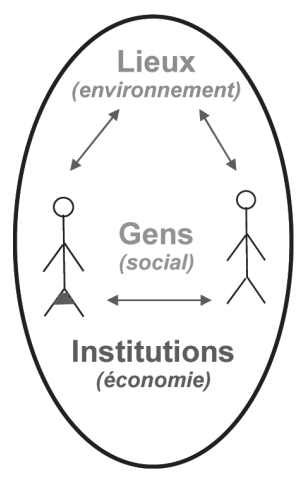

Un territoire est donc une sorte d'éco-système constitué par ces trois éléments constitutifs de base en «équilibre instable» ou en «déséquilibre dynamique» plus ou moins bien régulé. Dans leurs interactions, ces trois composantes génèrent des cultures locales que certains considèrent souvent comme la quatrième dimension-clé d'un territoire ou comme le quatrième pilier du développement soutenable qu'il faudrait pleinement prendre en compte. On retrouve dans ces constituants de base du territoire, les trois dimensions fondamentales que met donc en exergue le développement soutenable à savoir l'environnement (les lieux), le social (les gens), l'économique (les institutions), trois dimensions structurées par des logiques qu'il n’est pas aisé de rendre compatibles entre elles et dont la mise en cohérence, dépassant une simple régulation par le marché, apparaît comme relevant éminemment de la sphère politique. Cette mise en cohérence suppose un art de gouverner et de la mise en œuvre de pratiques démocratiques (Sen, 1999; World Bank, 2005).

\section{Les dynamiques de transformation des territoires urbains}

Dans le cas général, les transformations des différents constituants élémentaires du territoire conduisent à la rupture des cohérences construites antérieurement, chacune des composantes étant 
inscrites sur des trajectoires particulières et de manière relativement indépendante les unes des autres. Ces ruptures souvent qualifiées de «crise» au sens commun du terme, sont des déséquilibres dynamiques qui pourraient être assimilés à des «bifurcations» (Prigogine, 1999) ou encore à des «catastrophes» (Thom, 1983). S'ouvre alors une période plus ou moins longue de désajustements entre ces composantes, désajustements qui peuvent susciter des diagnostics empreints de jugements de valeur (on fait alors fréquemment référence aux quartiers anciens ou aux communautés stables du passé). Qu'en est-il en fait des transformations possibles des différentes composantes que sont les lieux, les gens et les institutions?

- Les lieux peuvent faire l'objet de processus de dévalorisation physique et technique, de processus d'obsolescence par rapport à d'autres espaces de la ville mieux équipés (confort des logements, architecture et agencement urbain), mieux achalandés (localisation, dessertes, services) et mieux valorisés symboliquement dans la hiérarchie des formes urbaines. En ce domaine, l'imaginaire urbain occupe une bonne place.

- Ces lieux peuvent être désertés par une partie des gens qui y vivaient, partis dans d'autres lieux de la ville et remplacés par des populations aux ressources, aux habitudes de vie et aux cultures différentes, des populations qui ont besoin de s'adapter à ce cadre de vie et d'adapter à leur mode de vie à ces lieux délaissés par leurs occupants précédents. Souvent ces nouveaux modes de vie ne sont pas en congruence avec les anciens lieux et les anciennes institutions, ils sont alors l'objet de stigmatisations par le voisinage et par les autorités.

- Les institutions existantes (activités économiques, services publics, organisations, associations) n'ont plus la même maîtrise sur les lieux et ne sont plus investies de la même manière par les nouveaux résidents. D'autres organisations se substituent à elles ou doivent leur être substituées. Cette transformation suppose du temps et un certain enracinement dans les lieux. Parfois les institutions existantes freinent ces nouvelles pratiques sociales et leur opposent des codes et des routines anciennes que celles-ci doivent contourner. Ces institutions génèrent des résistances qui peuvent pénaliser l'émergence de nouvelles mises en cohérence des lieux et des gens (manifestations régressives). On peut illustrer ces évolutions en indiquant les trajectoires sur lesquelles se déplacent tout ou partie de ces constituants élémentaires provoquant l'éclatement de la cohérence antérieure.

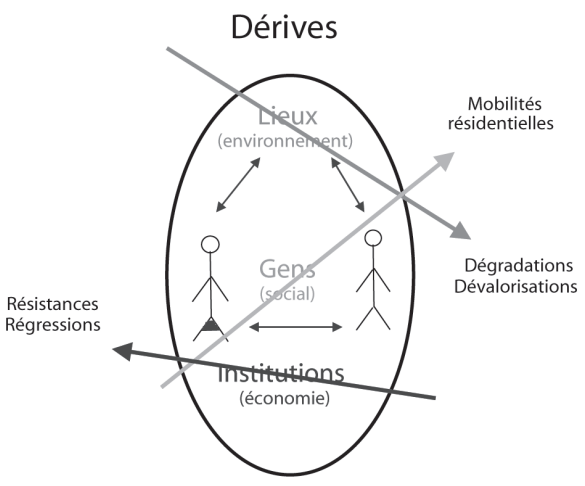

Les quelques logiques que l'on vient d'identifier sommairement provoquent un reclassement des différents territoires les uns par rapport aux autres au sein des villes et des régions urbaines. D'une période à l'autre, du fait de la transformation des villes, de nouveaux territoires urbanisés sont créés à l'initiative d'acteurs publics et privés, de responsables publics et de groupes d'intérêts. Ils participent et contribuent à ce repositionnement des territoires anciens les uns par rapport aux autres. Représenter la distribution de cette constellation de territoires n'est pas chose aisée tant sont nombreuses les situations possibles et discutables les représentations que l'on peut donner de leur hiérarchisation, celle-ci étant rarement fondée sur des données objectives mais manifestant plutôt des positionnements symboliques et parfois des partis pris idéologiques. A l'heure de la fragmentation sociale et spatiale accrue des villes, la logique dominante dans l'occupation du territoire ne consiste-t-elle pas à chercher à accéder aux espaces valorisés de la ville, quel qu'en soit le coût, au besoin en dévalorisant le territoire des autres (gestion de l'image et des représentations dans l'opinion) et 
en contribuant le moins possible aux solidarités métropolitaines nécessaires.

Pour illustrer cette complexité et essayer de comprendre la nature des dynamiques à l'œuvre, nous évoquerons ici, de manière simplifiée, les possibilités de repositionnement des territoires au sein de la hiérarchie urbaine (schéma ci-dessous). Imaginons la situation d'un territoire A0 et l'évolution possible de sa position dans la hiérarchie urbaine, soit sous l'effet de mécanismes marchands, soit consécutive à la mise en œuvre d'interventions publiques ou privées. Considérons ce territoire (A0) au temps t0, moment où ce territoire peut être considéré comme un espace d'élection de domicile. Ce territoire peut connaître bien des vicissitudes pour se retrouver au temps tn dans une position moins enviable (An) sur l'échelle des valeurs urbaines. A partir de cette situation au temps t n, plusieurs possibilités d'évolution peuvent se présenter pour ce territoire au temps tx.

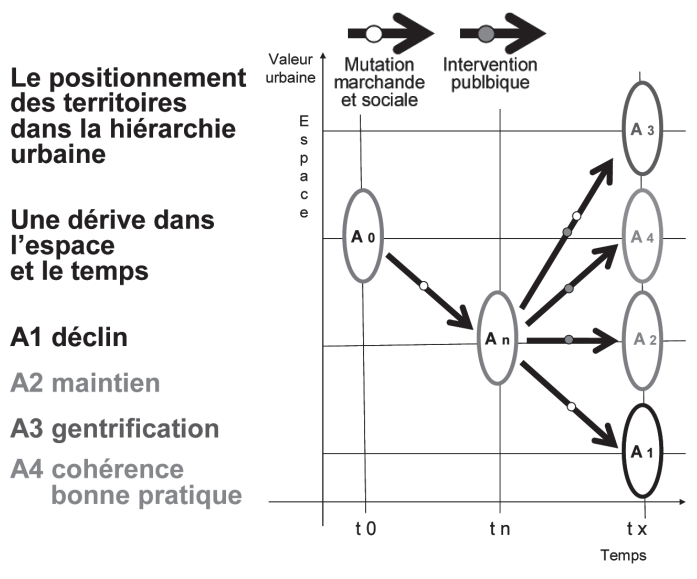

\section{Possibilité A1: poursuite du déclin}

L'évolution du territoire An se poursuit vers la possibilité Al en prolongement de la tendance passée, sans intervention publique ou avec une intervention publique minimum. Le territoire considéré poursuit sa dérive au sein de la structure urbaine: dégradation physique des lieux urbains et leur dévalorisation économique, départ des dernières populations ayant une capacité d'organiser et de représenter la communauté locale, renforcement du rôle de ce lieu dans l'accueil des populations les plus défavorisées de la région urbaine ou en provenance de l'extérieur (immigrants), impossibilité de renforcer, voire de maintenir les capacités des services présents dans le territoire (dégradation de la fonction commerciale, affaiblissement des services publics, dépérissement des organisations associatives et communautaires). En quelque sorte, ce territoire concentre l'ensemble des difficultés sociales. Il devient une sorte d'abcès de fixation dans la ville, un espace d'assignation à résidence. Parce qu'il joue ce rôle de lieu d'accueil des populations dont on ne veut pas ailleurs dans la ville, ce territoire devient une sorte d'opportunité pour de futures stratégies d'intervention: le niveau de la rente foncière et immobilière y est au plus bas et la rente potentielle y est maximum compte tenu de la dévalorisation généralisée de tous les biens ainsi que de la faible résistance possible des populations résidantes notamment lorsqu'il s'agit de populations sans droits civiques et politiques (étrangers) ou qui ne les exercent plus (abstention). Telle a été la dérive de nombreux quartiers anciens dans les centres villes partout en Europe. Telle a été la dérive de nombre de grands ensembles à partir des années soixante-dix et quatre-vingt au moment de la crise culturelle de la modernité urbaine et architecturale liée au début du processus de périurbanisation et au mouvement de reconquête des quartiers anciens (engouement pour le charme discret des vieilles poutres et des vieilles pierres).

\section{La possibilité A2: maintien de la situation}

Dans ce cas, le mouvement de dévalorisation est jugulé grâce essentiellement à l'intervention publique. On réussit à limiter le processus de dévalorisation du territoire considéré et celui-ci se maintient au niveau qu'il occupe aujourd'hui dans la hiérarchie des formes urbaines. L'autorité publique a été en mesure d'investir a minima dans les lieux (restauration de certains logements et de certains espaces publics, interventions conservatoires, parfois «cosmétiques» sur les façades et sur l'espace public) et elle a mis en œuvre des politiques d'assistance auprès des populations sans cependant provoquer de changements susceptibles d'inverser radicalement les tendances. Ce cas de figure est le plus fréquent dans les pays qui en sont au début de la mise en œuvre des politiques de régénération urbaine mais aussi dans ceux qui ont une certaine expérience en la matière (France, Grande Bretagne, Pays-Bas). 
La possibilité A3: processus de gentrification

Cette possibilité est celle qui correspond à une logique de revalorisation maximale de tout ou partie du territoire considéré. Cette revalorisation peut relever d'un processus «spontané» de nature privée (processus de reconquête des lieux selon une stratégie d'anticipation par rapport à la rente foncière et immobilière potentielle). Elle peut aussi être suscitée et favorisée par les stratégies explicites ou implicites de la puissance publique ou bien être accompagnée par elles. Parfois, mais plus rarement, il s'agit d'un processus de revalorisation qui a échappé à la maîtrise de la puissance publique (une revalorisation incontrôlée). Ce processus de régénération urbaine suppose, en général, la réunion de trois conditions: un lieu présentant de bonnes qualités patrimoniales (qualité architecturale et bon positionnement dans la structure urbaine) et donc une rente potentielle élevée, une faible résistance des populations résidantes face aux processus d'éviction (cas de personnes en situation illégale, d'occupants sans droits, ni titres, de squats), l'empathie ou la permissivité de la puissance publique. Cette logique débouche généralement sur un processus de «gentrification». En fait, dans ce cas de figure, il y a bien reconstruction d'une cohérence entre lieux-gens-institutions, mais ce ré-arrangement se fait en ignorant les gens qui résidaient-là auparavant. Ce n'est ni pour eux, ni avec eux, toujours à leur détriment et parfois en niant les caractéristiques des lieux et des institutions (changement $d$ 'atmosphère, mutations des commerces et des services, création de nouvelles instances de régulation). Ce processus va de pair avec des opérations urbaines généralement plus faciles à conduire qui portent sur le recyclage des friches industrielles urbaines (cf. brownfield). Ce cas de figure relativement fréquent dans les villes européennes, est généralement considéré comme une réussite par les autorités et les médias qui en assurent la diffusion alors qu'il s'agit, en fait, d'une fausse «bonne pratique» qui opère le plus souvent au détriment des résidents les plus défavorisés de ces territoire.

\section{La possibilité A4: cohérence et bonne pratique}

Ce dernier cas de figure correspond à ce qui est considéré comme un processus de régénération urbaine de qualité tel qu'il est décrit généralement par les guides professionnels et par les plaquettes d'information sur les projets. La régénération urbaine se réalise alors «par», «pour» et «avec» les résidents. C'est ce qu'on peut appeler une «bonne pratique». Ce résultat est bien évidemment le moins facile à obtenir et par conséquent, le moins fréquent dans les bilans et les statistiques. Il correspond à la construction d'une nouvelle mise en cohérence entre lieux, gens et institutions telle que nous l'évoquions précédemment en préservant les intérêts des gens déjà installés dans ces territoires. Cette bonne pratique que nous détaillerons dans le point suivant, suppose d'établir une astucieuse mise en correspondance entre territoires (lieuxgens-institutions) et projets d'intervention, une dialectique qui nécessite une bonne atmosphère ou un bon climat local (Marshall, 1890). Elle suppose aussi d'associer pleinement les résidents de ces territoires aux programmes de développement mis en œuvre ce qui nécessite de vraies stratégies d'empowerment des responsables des communautés locales en tenant compte tout particulièrement de la question du genre dès lors que ce sont les femmes qui y exercent le plus souvent les responsabilités.

\section{Les politiques intégrées de développement soutenable: le rôle politique majeur des villes et des régions urbaines.}

La mise en cohérence des constituants élémentaires des territoires et des communautés (lieux, gens, institutions) et de leurs transformations ne va pas de soi. Cette mise en cohérence est trop souvent présentée comme un mouvement spontané d'ajustement réciproque de ces composantes sur les marchés urbains (option «public choices»). $\mathrm{Si}$ elle relève souvent d'un tel ajustement spontané, on sait aussi qu'une telle combinatoire et un tel ajustement nécessitent pour s'établir l'intervention d'un pouvoir politique surplombant (option politique réformiste qui a été dominante dans les trente glorieuses de l'après-guerre). On sait toutefois que ces interventions publiques ne sont pas une garantie de reclassement optimum du territoire faisant l'objet d'interventions par rapport aux autres dans la ville. En fonction des objectifs poursuivis, objectifs plus ou moins explicites et explicités d'ailleurs, ces interventions peuvent prendre différentes formes 
et aboutir à des recompositions variées des composantes d'un territoire (Jacquier, 2006).

La régulation publique de cette recomposition des territoires urbains suppose de mobiliser les niveaux politico-institutionnels appropriés. Depuis les années quatre-vingt, le niveau local s'impose désormais pour penser et agir globalement car de plus en plus, il s'agit de «faire avec» ce qui existe déjà (Certeau, 1980) afin de coproduire la nouvelle combinatoire territoriale en s'appuyant sur la diversité des agents présents dans la ville. Dans l'Union Européenne, l'approche subsidiaire s'est progressivement imposée faisant primer un management et une régulation au plus près des enjeux locaux, les niveaux de gouvernement plus éloignés n'étant sollicités qu'en cas d'inefficience des instances de proximité. Cette approche subsidiaire rejoint la tendance des gouvernements centraux des dernières décennies qui vise à transférer nombre de compétences vers les pouvoirs locaux (décentralisation, dévolution, autonomisation). Désormais il est difficile de penser l'action publique en ces domaines en se focalisant uniquement sur le rôle des gouvernements centraux ou sur les grands enjeux internationaux et en passant sous silence l'action des instances intermédiaires que sont les villes, les régions, les organisations et les autorités locales ainsi que ceux qui vivent la ville au quotidien. Progressivement, même si cela est peu reconnu officiellement, les gouvernements centraux ont eu plutôt tendance à renvoyer vers les villes et les régions urbaines, la régulation des trois piliers du développement soutenable (Jacquier, 2005). C'est dans cette ville-là que peuvent s'imaginer et s'organiser concrètement les différents modes de coopérations nécessaires, anciens et récents, entre les acteurs socio-économiques et institutionnels: coopérations territoriales entre acteurs publics et privés, coopérations verticales entre niveaux institutionnels, coopérations horizontales ou transversales entre champs de compétences et services différents. Ces coopérations, nécessaires, souvent conflictuelles, aux frontières des domaines d'action traditionnels (métissages, hybridations), mais de mise en ouvre particulièrement délicate (cas de la coopération horizontale), sont des facteurs et des vecteurs essentiels de l'innovation au sein des sociétés et des économies contemporaines.

Ce rôle majeur des villes et des régions urbaines, la Commission Européenne l'avait d'ailleurs déjà fort bien identifié en 1997 et le rapport du Forum de Vienne de 1998 l'avait repris à son compte (Union Européenne 1997, 1999b). Ces rapports, outre le fait qu'ils s'appuyaient implicitement sur le triptyque du rapport Brundtland, insistaient particulièrement sur un quatrième volet, longtemps passé sous silence, à savoir «la gouvernance urbaine», en prônant l'élaboration et la mise en œuvre de politiques intégrées de développement urbain soutenable (notion reprise par la communication de la Commission de juillet 2006 et la Charte de Leipzig de mai 2007). Ces politiques intégrées de développement urbain soutenable sont rendues nécessaires par le passage de l'urbanisation extensive que tous les pays industrialisés ont connu à partir du milieu du $20^{\text {ème }}$ siècle à la recomposition des territoires déjà urbanisés: passage du «faire» la ville (à l'époque du boom de l'urbanisation) au «faire avec» la ville (avec les lieux, les gens, les institutions).

Les recherches effectuées sur ces politiques de développement ont permis de dégager quelques principes d'action communs s'articulant en une sorte «d'algorithme» du développement urbain soutenable et des bonnes pratiques de régénération urbaine. Cet algorithme peut être de quelque utilité pour penser les approches de prospective urbaine. Le cœur de cet algorithme est constitué par le jeu entre «territoire» (au sens où nous l'avons défini ci-dessus: lieux, gens, institutions) et «projet» : le territoire suggérant de nouvelles combinaisons de ses constituants, le projet étant en quête d'un territoire possible pour sa mise en œuvre. Cette dialectique «territoire-projet» est constitutive d'espaces de débats et génératrice de nouvelles combinaisons entre les acteurs qui y participent. Il y faut pour cela une certaine «atmosphère» (Marshall, 1890), un certain climat, un capital social et culturel. Les bonnes pratiques sont la résultante des relations toujours singulières entre ces trois constituants élémentaires de base que sont le territoire, le projet et l'atmosphère. La qualité de cette combinaison découle toujours du savoir-faire et du talent des professionnels mobilisés (d'où l'importance des ressources humaines, du recrutement et de la formation) ainsi que du leadership qui se manifeste sur ces territoires. Dans cette «dialectique» (territoire-projet-atmosphère) des coopérations entre acteurs peuvent alors se construire, coopérations qui prennent parfois un tour conflictuel 
(Perroux, 1990), mais qui sont constitutives de ce «partenariat» plus permanent qui est généralement recherché par ces nouvelles politiques urbaines et par les systèmes de gouvernance (cf. partenariat public privé). Cette coproduction en partenariat peut faire alors l'objet de «contrats» et de «conventions» stabilisant plus durablement la relation entre les acteurs, une contractualisation qui peut aboutir à une plus grande intégration des moyens respectifs des partenaires et, à terme, à une refonte de tout ou partie des organisations et de leurs territoires d'intervention pour permettre l'élaboration de nouveaux projets territoriaux. La «spirale algorithmique» pourrait alors se boucler à un niveau supérieur, en un renforcement de l'intégration des principes d'action et en une dynamique auto-entretenue de développement (intégration des politiques sectorielles, développement soutenable).

\section{Le développement soutenable et sa régulation Faire avec les territoires coopération - coproduction}
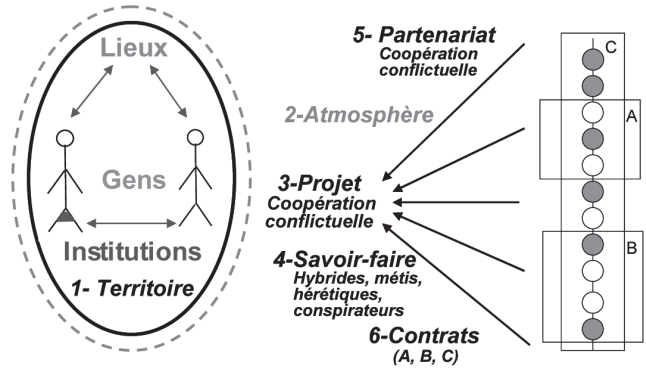

Ces approches intégrées de développement urbain soutenable sont de l'ordre d'un processus qui obéit à une logique ascendante (bottom-up) inverse de celle qui est généralement présentée dans les manuels de procédures. La logique de la procédure épouse dans les faits une logique descendante (top-down) où l'énoncé de l'ambition à atteindre («Projet intégré de développement urbain soutenable») ainsi que les conditions «contractuelles» et «partenariales» sont considérés comme des préalables de l'action. Ainsi, l'ordre de la procédure se déroule dans le schéma ci-dessus, du haut vers le bas (1-2-3-4-5-6-7) alors que l'ordre du processus épouse une logique du bas vers le haut (7-6-5-4-3-2-1) (schéma ci-dessus). En fait, dans la réalité, tout bon praticien doit savoir jouer habilement de la logique «processuelle» et de la logique «procédurale». La procédure s'appuie sur une organisation en appareils qui à l'avantage de la stabilité alors que le processus a à voir avec une organisation en réseaux, plus instable, mais qui a l'avantage de la flexibilité et de l'adaptabilité. Dans la pratique, il y a toujours coprésence de ces deux logiques processuelles et procédurales et une dialectique permanente entre appareils et réseaux (Lemieux, 1983) qui réclame un grand savoir-faire da le part des professionnels et des gestionnaires de projet (schéma ci-dessous).

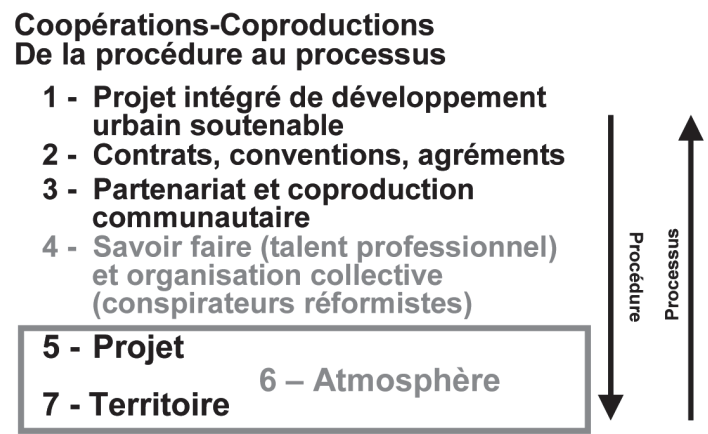

Une bonne pratique est fonction des relations entre "territoire-projet-atmosphère-savoir faire".

seul le savoir-faire est transférable.

Notons enfin que ce que l'on appelle bonne pratique (best practice en anglais) est au centre du processus à savoir à l'articulation entre «territoire / /atmosphère / projet / savoir-faire». L'innovation et la bonne pratique est la résultante de cet arrangement singulier de ces composantes-là, d'où la nécessité qu'il y a d'observer, d'analyser et d'accompagner ce qui le rend possible, notamment du côté des savoirs et des savoir-faire. En effet qu'en est-il de la fameuse transférabilité tant recherchée de cette bonne pratique? Mis à part les savoirs et les savoir-faire, aucune des autres composantes n'est vraiment transférable: ni le territoire, ni l'atmosphère, l'idée de projet, l'étant un peu mais alors on cela relève déjà du savoir-faire. Les savoirs et les savoir-faire doivent donc faire l'objet de toutes les attentions d'où, en Europe et avec le reste du monde, l'importance des programmes d'échange entre les villes, des programmes de formation et des programmes de recherche comparative.

\section{Conclusions}

Depuis des siècles, les villes ont joué un rôle essentiel dans le développement de leurs régions et de leurs contrées. Ce fait est connu et a été reconnu tout au long de l'histoire avant l'avènement des Etats-nations à partir du $19^{\text {ème }}$ siècle: voir les éco- 
nomies-monde (Braudel 1967), les communes médiévales face aux pouvoirs féodaux (Petit-Dutaillis, 1970), les villes de la Hanse (Dollinger, 1964), les cités-Etats italiennes, les villes du continent asiatique, etc. Dans le cadre des Etats-nations, elles ont encore joué un rôle socio-économique et culturel essentiel, tout particulièrement dans la construction européenne au cours de la seconde moitié du $20^{\text {ème }}$ siècle. Malheureusement, ce rôle politique a été systématiquement minoré ou est resté de l'ordre de l'implicite quand il n'a pas été dissimulé.

Depuis quelques décennies, on assiste à un renouveau des villes. Ainsi, les Etats-nations, parallèlement à leur affaiblissement dans des échanges mondialisés au profit des grandes instances internationales (Banque Mondiale, OMC) et continentales (UE), perdent de leur emprise sur les villes et les régions urbaines qui, dorénavant, tendent à jouer, un rôle-clé dans la régulation du développement soutenable en matière de compétitivité économique, de cohésion sociale et de précaution environnementale. En fait, c'est grâce aux villes et aux régions urbaines, ces acteurs collectifs innovateurs, que se réalise l'appropriation des grands objectifs de l'Union. Les services de la Commission ne s'y trompent pas même si, tactiquement, ils le taisent en traitant de plus en plus directement avec ces niveaux politiques décentralisés et en contournant les échelons centraux et déconcentrés de l'administration des Etats-nations. Nul doute que la Commission devrait formuler explicitement ce constat qui conditionne largement la réussite des engagements du sommet de Lisbonne pour 2010.

Toutefois, Etats et Commission ont du mal à se dégager d'une conception commune assez confuse de la réalité socio-économique et politique des villes. Villes et régions urbaines sont souvent considérées par eux comme des îlots de richesses dans un océan de misère rurale (ce que tendrait à montrer l'indicateur de PIB régional par tête) justifiant ainsi une distribution des ressources communautaires vers les régions «pauvres» périphériques alors que tout le monde sait que la distribution du revenu disponible par tête est tout autre et que l'irrigation du reste du territoire des Etats-membres se fait à partir de ces zones urbaines innovantes. En fait les villes et les régions urbaines sont des territoiresarchipels qui sont tout à la fois l'ombilic du monde (des territoires d'innovation) et sa périphérie la plus appauvrie (territoires où se concentrent les populations les plus démunies). C'est aussi particulièrement vrai en Europe et les résultats des référendums sur le traité constitutionnel en France et aux Pays-Bas l'ont largement montré. En part relative et surtout en part absolue notamment par rapport au monde rural, si c'est la partie riche des villes qui a voté «oui» au référendum, c'est la partie pauvre des villes qui a voté «non». Il y a peut-être là deux raisons suffisantes pour que les Etats membres et la Commission se soucient un peu plus d'orienter les ressources européennes vers les villes et les régions urbaines où se dessinent les profondes fractures des sociétés européennes, vers ces acteurs collectifs de l'innovation technologique et économique mais aussi vers ces acteurs collectifs de l'intégration et de la cohésion sociale et environnementale. C'est une évidence, mais les évidences ne sont pas suffisantes en politique, sauf à organiser un lobby puissant des villes et des régions urbaines relayé par le Parlement européen pour les transformer en arguments convaincants. Telle est peut-être aussi la condition d'un vrai programme de recherche européen portant sur la place et le rôle des villes et des régions urbaines dans la régulation politique du devenir du continent. 


\section{Bibliographie}

ARENDT, H. (1983), Condition de l'homme moderne, Calmann-Lévy.

BERQUE, Augustin (1999), «Milieu et architecture», in Yann Nussaume Tadao Andô et la question du milieu. Réflexions sur l'architecture et le paysage. Préface. Le Moniteur

BRAUDEL, F. (1967), Civilisation matérielle, Economie et Capitalisme XVe-XVIIe Siècle. Le Temps du Monde. Armand Colin.

CERTEAU, M. de, (1980), L'invention du quotidien. Arts de faire, 10/18 UGE, Paris.

COMMISSION EUROPÉENNE (2004) Un nouveau partenariat pour la cohésion: convergence, compétitivité, coopération. Février, Luxembourg, 186 p.

COMMISSION EUROPÉENNE (2006), La politique de cohésion et les villes : la contribution des villes et des agglomérations à la croissance et à l'emploi au sein des régions. Communication de la Commission au Conseil et au Parlement Européen, 13 juillet 2006, 15 p.

COMMISSION MONDIALE SUR L'ENVIRONNEMENT (1987). Notre avenir à tous. Rapport Brundtland Oxford University Press.

COMMISSION OF THE EUROPEAN COMMUNITIES (2006), Cohesion Policy and Cities: the Urban Contribution to Growth and Jobs in the Regions.- Communication from the Commission to the Council and Parliament, July.

COMMUNAUTÉS EUROPÉENNES (2004) Relever le défi. La stratégie de Lisbonne pour la croissance et l'emploi. Rapport du groupe de haut niveau présidé par Wim Kok, novembre, Luxembourg, 61 p.

DEBONNEUIL, M. FONTAGNE, L. (2003), Compétitivité. Conseil d'Analyse Economique, n ${ }^{\circ} 40$, Documentation Française.

DOLLINGER, P. (1964), La Hanse, XIIe-XVIIe siècles, Ed. Aubier, 1964.

EUROSTAT (2003), Revenu des ménages et produit intérieur brut dans les régions d'Europe. Statistiques en bref., Luxembourg.

HOBBES, T. (1921), Léviathan. Traité de la matière, de la forme et du pouvoir de la république ecclésiastique et civile, Paris, M. Giard (1651).

JACQUIER, C. (2005a) "On relationship between integrated policies for sustainable urban development and urban governance" in Beaumont J., Musterd S. Governance, Decentralization and the Rise of Local Participatory Democracy?. Tijdschrift voor Economische en Sociale Geografie Vol. 96, No. 4, the Royal Dutch Geographical Society KNAG, Blackwell Publishing, Oxford.

JACQUIER, C. (2006), "Can Distressed Urban Areas Become Growth Poles?" in Competitive Cities in the Global Economy, OECD Territorial Reviews. pp. 381-392.

KRUGMAN, P. (1991), Increasing Returns and Economic Geography, Journal of Political Economy, N 99(3), pp. 483 $-499$.

LEMIEUX, V. (1982) Réseaux et appareils: logique des systèmes et langage des graphes, Edisem, Québec.
MACHIAVEL, N. (1952), Sur la première décade de Tite-Live. Troisième livre, (Euvres complètes, La Pléiade, NRF (1526).

MARSHALL, A. (1890), Principles of Economics, Macmillan, London.

MEDARD, J-F. (1969), Communauté locale et organisation communautaire aux Etats-Unis.- Cahier de la Fondation Nationale des Sciences Politiques, Armand Colin.

OCDE (2002) La gouvernance pour le développement durable. Etude de cinq pays de l'OCDE, Paris, 284 p.

OECD (2001), Cities for Citizens. Improving Metropolitan Governance. Paris, 284 p.

PERROUX, F. (1964), L'économie du XXème siècle, PUF.

PERROUX, F. (1990) Dictionnaire économique et social, Hatier, Paris.

PETIT-DUTAILLIS C (1970).- Les communes françaises.- Albin Michel.

PRESIDENCE ALLEMANDE (2007), Charte de Leipzig sur la ville européenne durable, Conseil des ministres européen. (Leipzig Charter on Sustainable European Cities). Mai.

PRIGOGINE I, KONDEPUDI D (1999) Thermodyna-mique. Des moteurs thermiques aux structures dissipatives. Odile Jacob.

SEN, A. (1999), Democracy as a Universal Value - Journal of Democracy 10:3 National Endowment for Democracy and the Johns Hopkins University Press.

SIMMEL, G. (1981), Sociologie et épistémologie, Paris: PUF.

SOMBART, W. (1916), Der Moderne Kapitalismus, Duker Humbolt, Munchen.

THOM, René. (1983), Paraboles et catastrophes, Champ Flamarion.

TÖNNIES, F. (1944), Communauté et société. Catégories fondamentales de la sociologie pure, Paris, PUF (1887)

UNION EUROPÉENNE (1997), La question urbaine, orientations pour un débat.

UNION EUROPÉENNE (1999), Cadre d'action pour un développement urbain durable dans l'Union Européenne. Communication de la Commission.

UNION EUROPÉENNE (1999a) Schéma de développement de l'espace communautaire, Postdam, $94 \mathrm{p}$.

UNION EUROPÉENNE (2001). Rapport 2001 sur la compétitivité européenne.

WORLD BANK (2005) Governance Matters IV: Governance indicators for 1996-2004. Rapport Daniel Kaufmann, Aart Kraay and Massimo Mastruzzi, Washington, 61 p.

ZWIRN, H. P. (2006) Les systèmes complexes. Mathématiques et biologie, Odile Jacob. 